\title{
REFINED BOUNDS FOR THE EIGENVALUES OF THE KLEIN-GORDON OPERATOR
}

\author{
TÜRKAY YOLCU
}

(Communicated by Michael Hitrik)

\begin{abstract}
The aim of this article is twofold. First we establish sharper lower bounds for the sums of eigenvalues of $\left.(-\Delta)^{\frac{1}{2}}\right|_{D}$, the Klein-Gordon operator restricted to a bounded domain $D \subset \mathbb{R}^{d}$, than the bounds obtained in works by E. Harrell; S. Yıldırım Yolcu; and G. Wei, H. Sun, and L. Zeng. Then we study upper bounds for the sums of negative powers of the eigenvalues of $\left.(-\Delta)^{\frac{1}{2}}\right|_{D}$
\end{abstract}

\section{INTRODUCTION}

This article focuses on the estimates pertaining to the eigenvalues $\left\{\beta_{j}\right\}_{j=1}^{\infty}$ of the Klein-Gordon operator restricted to $D$ defined by

$$
\begin{aligned}
(-\Delta)^{\frac{1}{2}} w_{j} & =\beta_{j} w_{j} \text { in } D, \\
w_{j} & =0 \text { on } \partial D,
\end{aligned}
$$

where $D \subset \mathbb{R}^{d}$ is an open bounded domain and $d \geq 2$.

Note that from a probabilistic point of view $(-\Delta)^{\frac{1}{2}}$ restricted to $D$ is considered to be the generator of the Cauchy stochastic process killed upon exiting $\partial D$ and conveniently defined to be a non-local pseudo-differential operator in terms of Fourier transform as follows:

$$
\left.(-\Delta)^{\frac{1}{2}}\right|_{D} w=\mathcal{F}^{-1}\left[|\xi| \mathcal{F}\left[w \chi_{D}\right]\right]
$$

Here, $\mathbf{x} \mapsto \chi_{D}(\mathbf{x})$ designates the characteristic function defined to be 1 when $\mathbf{x} \in D$ and 0 when $\mathbf{x} \notin D$ and when $\mathcal{F}[w]$ and $\mathcal{F}^{-1}[w]$ denote the Fourier transform and inverse Fourier transform of a function $w: \mathbb{R}^{d} \rightarrow \mathbb{R}$ respectively:

$$
\mathcal{F}[w](\xi)=\frac{1}{(2 \pi)^{\frac{d}{2}}} \int_{\mathbb{R}^{d}} e^{-i \mathbf{x} \cdot \xi} w(\mathbf{x}) d \mathbf{x}, \quad \mathcal{F}^{-1}[w](x)=\frac{1}{(2 \pi)^{\frac{d}{2}}} \int_{\mathbb{R}^{d}} e^{i \mathbf{x} \cdot \xi} w(\xi) d \xi .
$$

For more details see [7, 10] and the references therein.

Let us first recall the analogue of Weyl's asymptotic formula for $\left.(-\Delta)^{\frac{1}{2}}\right|_{D}[2,3]$ :

$$
\beta_{k} \sim \sqrt{4 \pi} \frac{\left[\Gamma\left(1+\frac{d}{2}\right)\right]^{\frac{1}{d}}}{|D|^{\frac{1}{d}}} k^{\frac{1}{d}} \quad \text { as } \quad k \rightarrow \infty,
$$

Received by the editors February 6, 2012.

2010 Mathematics Subject Classification. Primary 35P15; Secondary 35P20.

Key words and phrases. Berezin-Li-Yau, counting function, eigenvalue, inequality, KleinGordon. 
where $|D|$ represents the volume of $D$ and $\Gamma(x)$ denotes the Gamma function $\Gamma(x)=$ $\int_{0}^{\infty} t^{x-1} e^{-t} d t$ for $x>0$. Moreover, by translating the set $D$ if necessary, we may also assume that the second moment $I(D)$ is given by

$$
I(D)=\int_{D}|\mathbf{x}|^{2} d \mathbf{x}
$$

In [3], the following Berezin-Li-Yau type bound inspired from [1,4] was obtained:

$$
\sum_{j=1}^{k} \beta_{j} \geq \sqrt{4 \pi} \frac{d}{d+1}\left(\frac{\Gamma\left(1+\frac{d}{2}\right)}{|D|}\right)^{\frac{1}{d}} k^{1+\frac{1}{d}} .
$$

Later, this result was improved in [7] by adding an additional term with $k^{1-\frac{1}{d}}$. Recently, adding another term with $k^{1-\frac{3}{d}}$ by using a different technique, it has been shown in 12 that the eigenvalues of Klein-Gordon operator satisfy

$$
\begin{aligned}
\sum_{j=1}^{k} \beta_{j} \geq & \sqrt{4 \pi} \frac{d}{d+1}\left(\frac{\Gamma\left(1+\frac{d}{2}\right)}{|D|}\right)^{\frac{1}{d}} k^{1+\frac{1}{d}}+\frac{1}{96 \sqrt{\pi}(d+1)} \frac{|D|^{1+\frac{1}{d}}}{I(D) \Gamma\left(1+\frac{d}{2}\right)^{\frac{1}{d}}} k^{1-\frac{1}{d}} \\
(1.5) & +\frac{(d-1)^{2}}{C(d) d(d+1)^{2} \pi^{\frac{3}{2}}} \frac{|D|^{2+\frac{3}{d}}}{I(D)^{2} \Gamma\left(1+\frac{d}{2}\right)^{\frac{3}{d}}} k^{1-\frac{3}{d}},
\end{aligned}
$$

where $C(2)=C(3)=49152$ and $C(d)=36864$ for $d \geq 4$.

One of our main objectives in this paper is to demonstrate a finer estimate than the estimate in (1.5) by exploiting the idea previously employed in [6,7,9, 11. More precisely, we shall prove the following result:

Theorem 1.1. For $d \geq 2, k \geq 1$, the eigenvalues $\left\{\beta_{j}\right\}_{j=1}^{\infty}$ of the Klein-Gordon operator $(-\Delta)^{\frac{1}{2}}$ restricted to $D$ satisfy

$$
\begin{aligned}
\sum_{j=1}^{k} \beta_{j} \geq & \sqrt{4 \pi} \frac{d}{d+1}\left(\frac{\Gamma\left(1+\frac{d}{2}\right)}{|D|}\right)^{\frac{1}{d}} k^{1+\frac{1}{d}}+\frac{1}{96 \sqrt{\pi}(d+1)} \frac{|D|^{1+\frac{1}{d}}}{I(D) \Gamma\left(1+\frac{d}{2}\right)^{\frac{1}{d}}} k^{1-\frac{1}{d}} \\
(1.6) \quad & +\frac{1}{576 \pi(d+1)} \frac{|D|^{\frac{3}{2}+\frac{2}{d}}}{I(D)^{\frac{3}{2}} \Gamma\left(1+\frac{d}{2}\right)^{\frac{2}{d}}} k^{1-\frac{2}{d}} .
\end{aligned}
$$

While it must be conceded that the estimate in (1.6) is arguably not the sharpest in theory, one can make it sharper presumably for higher dimensions by invoking the same technique employed here. For example, see Remark 4.1.

Let $N_{K}(z)$ denote the counting function which gives the number of eigenvalues $\beta_{j}$ less than or equal to $z$, namely,

$$
N_{K}(z)=\sup _{\beta_{j} \leq z}\{j\}=\sum_{j: \beta_{j} \leq z} 1 .
$$

Our second goal is to establish upper bounds for the sums of negative powers of eigenvalues and the counting function. Specifically, we shall prove the following estimates: 
Theorem 1.2. For $0<p<d$ and $d \geq 2$, the sums of negative powers of eigenvalues of the Klein-Gordon operator $(-\Delta)^{\frac{1}{2}}$ restricted to $D$ satisfy

$$
\sum_{j=1}^{k} \frac{1}{\beta_{j}^{p}} \leq(4 \pi)^{-\frac{p}{2}} \frac{d}{d-p}\left(\frac{|D|}{\Gamma\left(1+\frac{d}{2}\right)}\right)^{\frac{p}{d}} k^{1-\frac{p}{d}} .
$$

Corollary 1.3. The counting function $N_{K}$ for the Klein-Gordon operator restricted to $D$ has the following upper bound for $z \geq 0$ :

$$
N_{K}(z) \leq \frac{(d+1)^{d}}{d^{d}} \frac{1}{(4 \pi)^{\frac{d}{2}}} \frac{|D|}{\Gamma\left(1+\frac{d}{2}\right)} z^{d} .
$$

See 9] for further results on tiling domains $D$ in $\mathbb{R}^{d}$.

\section{REVIEW OF WELL-KNOWN FACTS}

This section contains essential definitions and tools previously developed in [7, and so it actually provides a springboard for us to establish the estimates in (1.6) and (1.7). Throughout this article, $B_{R}(\mathbf{y}):=\left\{\mathbf{x} \in \mathbb{R}^{d}:|\mathbf{x}-\mathbf{y}| \leq R\right\}$ represents the ball of radius $R$ centered at $\mathbf{y}$ in $\mathbb{R}^{d}$ and $w_{d}$ denotes the volume of a $d$ dimensional unit ball $B_{1}(\mathbf{x})$ in $\mathbb{R}^{d}$ given by

$$
w_{d}=\frac{\pi^{\frac{d}{2}}}{\Gamma\left(1+\frac{d}{2}\right)} .
$$

In this setting, the surface area of the unit ball $B_{1}$ in $\mathbb{R}^{d}$ is $d w_{d}$.

We remark that the eigenvalues $\left\{\beta_{j}\right\}_{j=1}^{\infty}$ including multiplicities can be sorted as 3

$$
0<\beta_{1} \leq \beta_{2} \leq \cdots \leq \beta_{k} \leq \cdots \rightarrow \infty \text { as } k \rightarrow \infty .
$$

Let us now review some well-known properties of the eigenfunctions of the KleinGordon operator. That the set of eigenfunctions $\left\{w_{j}\right\}_{j=1}^{\infty}$ is an orthonormal set in $L^{2}(D)$ results in the fact that the set of Fourier transforms $\left\{\hat{w}_{j}\right\}_{j=1}^{\infty}$ of $\left\{w_{j}\right\}_{j=1}^{\infty}$ also forms an orthonormal set in $L^{2}\left(\mathbb{R}^{d}\right)$ by using Plancherel's theorem. To ease the notation in what follows we set

$$
W_{k}(\xi):=\sum_{j=1}^{k}\left|\hat{w}_{j}(\xi)\right|^{2}=\sum_{j=1}^{k}\left|\frac{1}{(2 \pi)^{\frac{d}{2}}} \int_{D} e^{-i \mathbf{x} \cdot \xi} w_{j}(\mathbf{x}) d \mathbf{x}\right|^{2} \geq 0 .
$$

Notice that the integral is taken over $D$ instead of $\mathbb{R}^{d}$ because the support of $w_{j}$ is $D$.

The following crucial properties of $W_{k}$ are extracted from Section 2 of [7].

Lemma 2.1 ([7]). The function $W_{k}$ defined by (2.3) satisfies

$$
\begin{gathered}
\int_{\mathbb{R}^{d}} W_{k}(\xi) d \xi=k, \\
W_{k}(\xi) \leq \frac{|D|}{(2 \pi)^{d}}, \quad \xi \in \mathbb{R}^{d}, \\
\int_{\mathbb{R}^{d}}|\xi| W_{k}(\xi) d \xi=\sum_{j=1}^{k} \beta_{j},
\end{gathered}
$$




$$
\left|\nabla W_{k}(\xi)\right| \leq m_{K}:=2(2 \pi)^{-d} \sqrt{|D| I(D)} .
$$

Let $r$ be the number such that $|D|=w_{d} r^{d}$. Since

$$
I(D) \geq \int_{B_{r}(0)}|\mathbf{x}|^{2} d \mathbf{x}=\frac{d w_{d}}{d+2} r^{d+2}=\frac{d}{d+2} w_{d}^{-\frac{2}{d}}|D|^{\frac{d+2}{d}},
$$

we have

$$
m_{K}=2(2 \pi)^{-d} \sqrt{|D| I(D)} \geq \frac{|D|^{\frac{d+1}{d}}}{(2 \pi)^{d} w_{d}^{\frac{1}{d}}} .
$$

Moreover, supposing that $W_{k}^{*}(\xi)$ denotes the decreasing radial rearrangement of $W_{k}(\xi)$, by approximating $W_{k}$, we may infer that there exists a real valued absolutely continuous function $\varrho_{k}:[0, \infty) \rightarrow\left[0,(2 \pi)^{-d}|D|\right]$ such that

$$
W_{k}^{*}(\xi)=\varrho_{k}(|\xi|) .
$$

Also, we define the distribution function $\nu_{k}$ by

$$
\nu_{k}(s):=\left|\left\{W_{k}(\xi)>s\right\}\right|=\left|\left\{W_{k}^{*}(\xi)>s\right\}\right| .
$$

Then, $\nu_{k}\left(\varrho_{k}(t)\right)=w_{d} t^{d}$. Indeed,

$$
\nu_{k}\left(\varrho_{k}(t)\right)=\left|\left\{W_{k}^{*}(\xi)>\varrho_{k}(t)\right\}\right|=|\{\xi:|\xi|<t\}|=\left|B_{t}(0)\right|=w_{d} t^{d} .
$$

Invoking the coarea formula in view of (2.5), we have

$$
\nu_{k}(s)=\int_{s}^{\infty} \int_{\left\{W_{k}^{-1}(t)\right\}} \frac{1}{\left|\nabla W_{k}\right|} d \sigma d t=\int_{s}^{(2 \pi)^{-d}|D|} \int_{\left\{W_{k}=t\right\}} \frac{1}{\left|\nabla W_{k}\right|} d \sigma d t
$$

where $\sigma$ is the $(d-1)$ dimensional Hausdorff measure. Let us consider $t \geq 0$ such that $\varrho_{k}^{\prime}(t)<0$. Then the isoperimetric inequality,

$$
\sigma(\partial D) \geq d w_{d}^{\frac{1}{d}}|\bar{D}|^{\frac{d-1}{d}}, \quad D \subset \mathbb{R}^{d},
$$

results in

$$
\begin{aligned}
\frac{d w_{d} t^{d-1}}{\varrho_{k}^{\prime}(t)} & =\nu_{k}^{\prime}\left(\varrho_{k}(t)\right) \\
& =-\int_{\left\{W_{k}=\varrho_{k}(t)\right\}} \frac{1}{\left|\nabla W_{k}\right|} d \sigma \\
\text { by (2.7) } & \leq-\frac{1}{m_{K}} \sigma\left(\left\{W_{k}=\varrho_{k}(t)\right\}\right) \\
& \leq-\frac{1}{m_{K}} d w_{d}^{\frac{1}{d}} \nu_{k}\left(\varrho_{k}(t)\right)^{\frac{d-1}{d}} \\
& =-\frac{1}{m_{K}} d w_{d} t^{d-1} .
\end{aligned}
$$

This inequality combined with $\varrho_{k}^{\prime} \leq 0$ simply means

$$
0 \leq-\varrho_{k}^{\prime}(t) \leq m_{K} .
$$




\section{Proof of Theorem 1.1}

Our method of proof has been previously explored in several articles [6 6 , 10, 11] with crucial differences. As we shall see later, the main ingredient in the proof of the refined lower bound in (1.6) that we want to prove is the following elementary inequality:

Lemma 3.1. For an integer $d \geq 2$ and positive real numbers $s, t$ we have the following inequality:

$$
d t^{d+1}-(d+1) t^{d} s+s^{d+1}-\left(s^{d-1}+2 t s^{d-2}\right)(t-s)^{2} \geq 0 .
$$

Proof. Using induction on $d \geq 2$, we first observe that

$$
d x^{d+1}-(d+1) x^{d}+1-(1+2 x)(x-1)^{2}=(x-1)^{2} \sum_{n=1}^{d-2}(n+2) x^{n+1} .
$$

Note that when $d=2$, the right-hand side of (3.2) is 0 . Therefore, noticing that the right-hand side of (3.2) is nonnegative, setting $x=t / s$, and rewriting (3.2), we conclude (3.1).

The following key result, inspired from the observation in [6], is very substantial because it helps us make a connection between two integrals to be considered in (3.10). A short proof is given so that the exposition will be self-contained.

Lemma 3.2. Suppose that $\tau:[0, \infty) \rightarrow[0,1]$ such that

$$
0 \leq \tau \leq 1 \quad \text { and } \quad \int_{0}^{\infty} \tau(t) d t=1 .
$$

Then, there exists $\delta \geq 0$ so that

$$
\int_{\delta}^{\delta+1} t^{d} d t=\int_{0}^{\infty} t^{d} \tau(t) d t
$$

Also, we infer that $\tau$ satisfies

$$
\int_{\delta}^{\delta+1} t^{d+1} d t \leq \int_{0}^{\infty} t^{d+1} \tau(t) d t
$$

Proof. Let us consider $\psi_{d}:[0, \infty) \rightarrow(0, \infty)$ defined by

$$
\psi_{d}(z)=\frac{(z+1)^{d+1}-z^{d+1}}{d+1}=\int_{z}^{z+1} t^{d} d t
$$

First notice that

$$
\left(t^{d}-1\right)\left(\tau(t)-\chi_{[0,1]}(t)\right) \geq 0, \quad t \in[0, \infty) .
$$

Integrating (3.6) from 0 to $\infty$ gives

$$
\int_{0}^{\infty} t^{d} \tau(t) d t \geq \frac{1}{d+1}=\psi_{d}(0)
$$

Since $\psi_{d}$ is continuous and non-decreasing and $\psi_{d}(z) \rightarrow \infty$ as $z \rightarrow \infty$, the Intermediate Value Theorem provides us with the existence of $\delta \geq 0$ such that

$$
\psi_{d}(\delta)=\int_{0}^{\infty} t^{d} \tau(t) d t,
$$

which gives (3.4). To see (3.5), we now consider the polynomial

$$
\phi_{d}(t)=t^{d+1}-a_{1} t^{d}+a_{2}=t^{d}\left(t-a_{1}\right)+a_{2},
$$


where $a_{1} \geq 0$ and $a_{2} \geq 0$ are chosen so that $\phi_{d}(\delta)=0$ and $\phi_{d}(\delta+1)=0$ and $\phi_{d}$ remains negative on $(\delta, \delta+1)$ and positive on $[0, \infty) \backslash[\delta, \delta+1]$. It is immediate to observe that

$$
\phi_{d}(t)\left(\chi_{[\delta, \delta+1]}(t)-\tau(t)\right) \leq 0 \quad \text { on } \quad[0, \infty) .
$$

Integration of (3.7) on $[0, \infty)$ together with (3.3) gives

$$
\int_{\delta}^{\delta+1} t^{d+1} d t \leq \int_{0}^{\infty} t^{d+1} \tau(t) d t-a_{1}\left(\int_{0}^{\infty} t^{d} \tau(t) d t-\int_{\delta}^{\delta+1} t^{d} d t\right)
$$

which together with (3.4) yields the inequality in (3.5). This finishes the proof.

Armed with Lemma 3.1 and Lemma 3.2, we are ready to obtain the following core estimate, which can be viewed as a precursor to the proof of Theorem 1.1 .

Lemma 3.3. Assume that (2.4) $-(2.6)$ hold. For $d \geq 2$, any real number $\epsilon \in(0,1]$ and each integer $k \geq 1$, we have the following inequality:

$$
\begin{aligned}
\sum_{j=1}^{k} \beta_{j} \geq & \frac{d}{d+1} w_{d}^{-\frac{1}{d}} \varrho_{k}(0)^{-\frac{1}{d}} k^{1+\frac{1}{d}}+\frac{\epsilon}{12(d+1)} m_{K}^{-2} w_{d}^{\frac{1}{d}} \varrho_{k}(0)^{2+\frac{1}{d}} k^{1-\frac{1}{d}} \\
& +\frac{\epsilon}{18(d+1)} m_{K}^{-3} w_{d}^{\frac{2}{d}} \varrho_{k}(0)^{3+\frac{2}{d}} k^{1-\frac{2}{d}} .
\end{aligned}
$$

Proof. Consider the decreasing, absolutely continuous function $\varrho_{k}:[0, \infty) \rightarrow(0, \infty)$ defined by (2.9). We know that $0 \leq-\varrho_{k}^{\prime}(t) \leq m_{K}$ for $t \geq 0$, where $m_{K}>0$ is given by (2.8). Since $\varrho_{k}(0)>0$ due to (2.3) , let us first define

$$
T_{k}(t):=\frac{1}{\varrho_{k}(0)} \varrho_{k}\left(\frac{\varrho_{k}(0)}{m_{K}} t\right) .
$$

Note that $T_{k}$ is positive, $T_{k}(0)=1$ and $0 \leq-T_{k}^{\prime}(t) \leq 1$. To simplify the notation, we also set $\tau_{k}(t):=-T_{k}^{\prime}(t)$ for $t \geq 0$. Hence, $0 \leq \tau_{k}(t) \leq 1$ for $t \geq 0$ and

$$
\int_{0}^{\infty} \tau_{k}(t) d t=T_{k}(0)=1
$$

Now, set

$$
\alpha_{k}=\int_{0}^{\infty} t^{d-1} T_{k}(t) d t \quad \text { and } \quad \gamma_{k}=\int_{0}^{\infty} t^{d} T_{k}(t) d t
$$

Assume that $\gamma_{k}<+\infty$. Suppose that $t^{d+1} T_{k}(t) \rightarrow L>0$ as $t \rightarrow \infty$. Then for any $0<a<L$ we can find a finite number $N>0$ such that

$$
0<\frac{L-a}{t}<t^{d} T_{k}(t)<\frac{L+a}{t}
$$

for any $t>N$. Therefore, using (3.11) we arrive at

$$
\infty=\int_{N}^{\infty} t^{d} T_{k}(t) d t \leq \int_{0}^{\infty} t^{d} T_{k}(t) d t<\infty,
$$

which is a contradiction, as $0 \leq \gamma_{k}<\infty$. Thus, $t^{d+1} T_{k}(t) \rightarrow 0$ as $t \rightarrow \infty$. Moreover, it is not difficult to observe that $t^{d} T_{k}(t) \rightarrow 0$ as $t \rightarrow \infty$ as well. Thus, using integration by parts, we obtain

$$
\int_{0}^{\infty} t^{d} \tau_{k}(t) d t=\alpha_{k} d \quad \text { and } \quad \int_{0}^{\infty} t^{d+1} \tau_{k}(t) d t=\gamma_{k}(d+1) .
$$


Now Lemma 3.2 allows us to conclude that there exists $\delta \geq 0$ such that

$$
\int_{\delta}^{\delta+1} t^{d} d t=\alpha_{k} d
$$

and

$$
\int_{\delta}^{\delta+1} t^{d+1} d t \leq \int_{0}^{\infty} t^{d+1} \tau_{k}(t) d t=\gamma_{k}(d+1)
$$

Using Jensen's inequality we see that

$$
\alpha_{k} d=\int_{\delta}^{\delta+1} t^{d} d t \geq\left(\int_{\delta}^{\delta+1} t d t\right)^{d} \geq\left(\int_{0}^{1} t d t\right)^{d}=\frac{1}{2^{d}} .
$$

Notice that (3.1) gives the key inequality in the proof of this lemma. Indeed, integrating (3.1) in $t$ from $\delta$ to $\delta+1$ we obtain

$$
\begin{aligned}
\int_{\delta}^{\delta+1} t^{d+1} d t \geq & \frac{d+1}{d} s \int_{\delta}^{\delta+1} t^{d} d t-\frac{1}{d} s^{d+1}+\frac{1}{d} s^{d-1} \int_{\delta}^{\delta+1}(t-s)^{2} d t \\
& +\frac{2}{d} s^{d-2} \int_{\delta}^{\delta+1} t(t-s)^{2} d t
\end{aligned}
$$

Now, we first note that for any $s \geq 1 / 2$ and $\delta \geq 0$, we have

$$
\begin{aligned}
& \int_{\delta}^{\delta+1}(t-s)^{2} d t \geq \int_{s-\frac{1}{2}}^{s+\frac{1}{2}}(t-s)^{2} d t=\frac{1}{12} \\
& \int_{\delta}^{\delta+1} t(t-s)^{2} d t=s^{2} \int_{\delta}^{\delta+1} t d t+\int_{\delta}^{\delta+1} t^{2}(t-2 s) d t \\
& \geq s^{2} \int_{0}^{1} t d t+\int_{0}^{1} t^{2}(t-2 s) d t \\
&=\frac{1}{2} s^{2}-\frac{2}{3} s+\frac{1}{4} \geq \frac{1}{36} .
\end{aligned}
$$

Since $\left(\alpha_{k} d\right)^{\frac{1}{d}} \geq 1 / 2$ due to (3.14), setting $s=\left(\alpha_{k} d\right)^{\frac{1}{d}}$ and using (3.12), (3.13), (3.16) and (3.17), we deduce that (3.15) leads to

$$
(d+1) \gamma_{k} \geq\left(\alpha_{k} d\right)^{1+\frac{1}{d}}+\frac{1}{12 d}\left(\alpha_{k} d\right)^{1-\frac{1}{d}}+\frac{1}{18 d}\left(\alpha_{k} d\right)^{1-\frac{1}{d}} .
$$

Therefore, simplifying the terms we end up with

$$
\gamma_{k} \geq \frac{1}{d+1}\left(\alpha_{k} d\right)^{1+\frac{1}{d}}+\frac{\varepsilon}{12 d(d+1)}\left(\alpha_{k} d\right)^{1-\frac{1}{d}}+\frac{\varepsilon}{18 d(d+1)}\left(\alpha_{k} d\right)^{1-\frac{1}{d}},
$$

which holds true for any $0<\varepsilon \leq 1$. Using (2.4) we get

$$
k=\int_{\mathbb{R}^{d}} W_{k}(\xi) d \xi=\int_{\mathbb{R}^{d}} W_{k}^{*}(\xi) d \xi=d w_{d} \int_{0}^{\infty} t^{d-1} \varrho_{k}(t) d t .
$$

Moreover, since the map $\xi \mapsto|\xi|$ is radial and increasing, by (2.6), we obtain that

$$
\sum_{j=1}^{k} \beta_{j}=\int_{\mathbb{R}^{d}}|\xi| W_{k}(\xi) d \xi \geq \int_{\mathbb{R}^{d}}|\xi| W_{k}^{*}(\xi) d \xi=d w_{d} \int_{0}^{\infty} t^{d} \varrho_{k}(t) d t .
$$


Substitution of (3.9) into (3.10) yields

$$
\begin{aligned}
\alpha_{k} & =\int_{0}^{\infty} t^{d-1} T_{k}(t) d t=\frac{m_{K}^{d}}{\varrho_{k}(0)^{d+1}} \int_{0}^{\infty} t^{d-1} \varrho_{k}(t) d t=\frac{m_{K}^{d}}{\varrho_{k}(0)^{d+1}} \frac{k}{d w_{d}}, \\
\gamma_{k} & =\int_{0}^{\infty} t^{d} T_{k}(t) d t=\frac{m_{K}^{d+1}}{\varrho_{k}(0)^{d+2}} \int_{0}^{\infty} t^{d} \varrho_{k}(t) d t \leq \frac{m_{K}^{d+1}}{\varrho_{k}(0)^{d+2}} \frac{\sum_{j=1}^{k} \beta_{j}}{d w_{d}},
\end{aligned}
$$

which combined with (3.20) and (3.21) turns (3.19) into (3.8), as claimed. Note that if $\gamma_{k}=+\infty$, then (3.8) is always true; there is nothing to prove.

\section{Proof of MAin Results}

This section reports the proofs of our main results.

4.1. Proof of Theorem 1.1. Now we are ready to prove Theorem 1.1 by using Lemmas 3.1 , 3.2 and 3.3 .

Proof. Our point of departure is to minimize (3.8) over $\varrho_{k}(0)$. To this end, let us set $x=\varrho_{k}(0)>0$. By (2.5) we know that $0<x \leq(2 \pi)^{-d}|D|$. Here, the clincher is to separately consider the monotonicity of two functions, $F_{1}, F_{2}:\left(0,(2 \pi)^{-d}|D|\right] \rightarrow$ $(0, \infty)$ defined by

$$
F_{1}(x)=\frac{c_{1}}{2} x^{-\frac{1}{d}}+c_{2} x^{2+\frac{1}{d}}, \quad F_{2}(x)=\frac{c_{1}}{2} x^{-\frac{1}{d}}+c_{3} x^{3+\frac{2}{d}} .
$$

Simply differentiating $F_{1}$ and $F_{2}$, we see that $F_{1}(x)$ is decreasing when $0<x \leq$ $\left(\frac{c_{1}}{(4 d+2) c_{2}}\right)^{\frac{d}{2 d+2}}$, while $F_{2}(x)$ is decreasing when $0<x \leq\left(\frac{c_{1}}{(6 d+4) c_{3}}\right)^{\frac{d}{3 d+3}}$. Choosing

$$
c_{1}=\frac{d k^{1+\frac{1}{d}}}{(d+1) w_{d}^{\frac{1}{d}}}, \quad c_{2}=\frac{\varepsilon w_{d}^{\frac{1}{d}} k^{1-\frac{1}{d}}}{12(d+1) m_{K}^{2}}, \quad c_{3}=\frac{\varepsilon w_{d}^{\frac{2}{d}} k^{1-\frac{2}{d}}}{18(d+1) m_{K}^{3}},
$$

we particularly observe that $x \mapsto\left(F_{1}+F_{2}\right)(x)$ is decreasing on $\left(0,(2 \pi)^{-d}|D|\right]$ when we have

$$
\frac{|D|}{(2 \pi)^{d}} \leq \min \left\{\left(\frac{6 m_{K}^{2} d k^{\frac{2}{d}}}{\varepsilon(2 d+1) w_{d}^{\frac{2}{d}}}\right)^{\frac{d}{2 d+2}},\left(\frac{9 m_{K}^{3} d k^{\frac{3}{d}}}{\varepsilon(3 d+2) w_{d}^{\frac{3}{d}}}\right)^{\frac{d}{3 d+3}}\right\}
$$

for any $k \geq 1$. In other words, in view of the lower bound $m_{K} \geq(2 \pi)^{-d} w_{d}^{-\frac{1}{d}}|D|^{\frac{d+1}{d}}$ given in (2.8) and the definition of $w_{d}$ in (2.1), we may take $\varrho_{k}(0)=(2 \pi)^{-d}|D|$ when we have

$$
\varepsilon \leq \min _{d \geq 2}\left\{\Theta_{1}(d), \Theta_{2}(d)\right\}
$$

where

$$
\Theta_{1}(d)=\frac{24 d}{2 d+1}\left[\Gamma\left(1+\frac{d}{2}\right)\right]^{\frac{4}{d}}, \quad \Theta_{2}(d)=\frac{72 d}{3 d+2}\left[\Gamma\left(1+\frac{d}{2}\right)\right]^{\frac{6}{d}} .
$$

Note that $\Theta_{1}(d)$ and $\Theta_{2}(d)$, running over integers greater than or equal to 2 , assume their minimum at $d=2$ with values $\Theta_{1}(2)=9.6$ and $\Theta_{2}(2)=18$, respectively (see Figure 1). With this observation at hand, we are allowed to replace $\varrho_{k}(0)$ with $|D|(2 \pi)^{-d}$ in (3.8) when we set

$$
\varepsilon=\min \left\{1, \Theta_{1}(d), \Theta_{2}(d)\right\}=1 .
$$



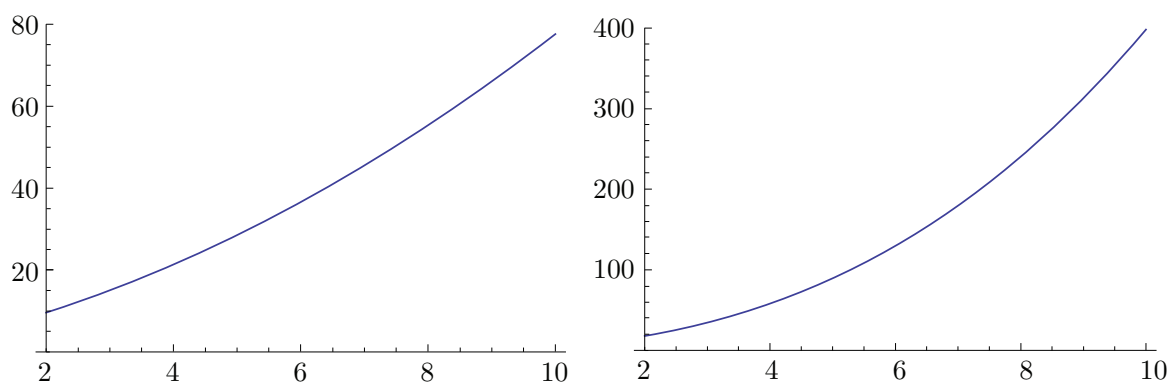

Figure 1. Graphs of $\Theta_{1}(d)$ and $\Theta_{2}(d)$ versus $d \geq 2$ respectively.

Thus, substitution of $m_{K}=2(2 \pi)^{-d} \sqrt{|D| I(D)}$ given in (2.8) together with $\varrho_{k}(0)=$ $|D|(2 \pi)^{-d}$ turns (3.8) into (1.6) as claimed.

Remark 4.1. Rewriting (3.2) as

$$
d x^{d+1}-(d+1) x^{d}+1=(x-1)^{2} \sum_{n=-1}^{d-2}(n+2) x^{n+1}
$$

and plugging in $x=t / s$, we get

$$
d t^{d+1}=\left((d+1) t^{d} s-s^{d+1}\right)+(t-s)^{2} \Sigma(d, s, t),
$$

where

$$
\Sigma(d, s, t)=\sum_{n=-1}^{d-2}(n+2) t^{n+1} s^{d-n-2} .
$$

The first terms on the left and the right of (4.6) with the same setting yield (1.4). The second term on the right of (4.6) gives an improved estimate of similar kinds, which seems to be practical for a fixed dimension $d \geq 3$ or numerical computations.

4.2. Proof of Theorem 1.2 and Corollary 1.3. The following proof is inspired from the proof of the Berezin-Li-Yau inequality in [4,11. An analogous proof is also exploited in [13] by means of the bathtub principle [5].

Proof. Assuming the properties (2.4)-(2.6), we first define

$$
V_{k}(\xi)=\frac{|D|}{(2 \pi)^{d}} \chi_{B_{R_{k}}(0)}(\xi), \quad R_{k}=\left(\frac{(d-p)(2 \pi)^{d}\left(\sum_{j=1}^{k} \frac{1}{\beta_{j}^{p}}\right)}{d w_{d}|D|}\right)^{\frac{1}{d-p}}
$$

so that

$$
\int_{\mathbb{R}^{d}} \frac{V_{k}(\xi)}{|\xi|^{p}} d \xi=\sum_{j=1}^{k} \frac{1}{\beta_{j}^{p}} .
$$

Since $\left|\hat{w}_{j}(\xi)\right|^{2} d \xi$ is a probability measure on $\mathbb{R}^{d}$ and $x \mapsto x^{-p}$ is convex for $x>0$ and $p>0$, employing Jensen's inequality and (2.3), we obtain that

$$
\sum_{j=1}^{k} \frac{1}{\beta_{j}^{p}}=\sum_{j=1}^{k}\left(\int_{\mathbb{R}^{d}}|\xi|\left|\hat{w}_{j}(\xi)\right|^{2} d \xi\right)^{-p} \leq \int_{\mathbb{R}^{d}} \frac{W_{k}(\xi)}{|\xi|^{p}} d \xi
$$


Now observe that

$$
\left(\frac{1}{|\xi|^{p}}-\frac{1}{R_{k}^{p}}\right)\left(W_{k}(\xi)-V_{k}(\xi)\right) \leq 0 .
$$

Integrating (4.10) on $\mathbb{R}^{d}$ and using (4.8) we arrive at

$$
\frac{1}{R_{k}^{p}} \int_{\mathbb{R}^{d}}\left(W_{k}(\xi)-V_{k}(\xi)\right) d \xi \geq \int_{\mathbb{R}^{d}} \frac{\left(W_{k}(\xi)-V_{k}(\xi)\right)}{|\xi|^{p}} d \xi \geq 0,
$$

from which it follows that

$$
\int_{\mathbb{R}^{d}} W_{k}(\xi) d \xi \geq \int_{\mathbb{R}^{d}} V_{k}(\xi) d \xi
$$

Thus, by (2.4), we obtain

$$
k \geq \int_{\mathbb{R}^{d}} V_{k}(\xi) d \xi=\frac{|D|}{(2 \pi)^{d}} d w_{d}\left(\frac{R_{k}^{d}}{d}\right) .
$$

Substituting $w_{d}$ given by (2.1) and $R_{k}$ given by (4.7) into (4.12) and rearranging the terms, we deduce the inequality in (1.7).

Now, we are ready to prove Corollary 1.3 .

Proof. To see (1.8) we consider $z \in\left[\beta_{k}, \beta_{k+1}\right)$ so that $N_{K}(z)=k$. Now we use (2.2), take $p=1$ in (1.7) and observe that

$$
\frac{1}{z} \leq \frac{1}{k} \frac{k}{\beta_{k}} \leq \frac{1}{k} \sum_{j=1}^{k} \frac{1}{\beta_{j}} \leq(4 \pi)^{-\frac{1}{2}} \frac{d}{d-1}\left(\frac{|D|}{\Gamma\left(1+\frac{d}{2}\right)}\right)^{\frac{1}{d}} k^{-\frac{1}{d}},
$$

which together with $k=N_{K}(z)$ leads to the following inequality:

$$
N_{K}(z) \leq \frac{d^{d}}{(d-1)^{d}} \frac{1}{(4 \pi)^{\frac{d}{2}}} \frac{|D|}{\Gamma\left(1+\frac{d}{2}\right)} z^{d} .
$$

On the other hand, using (1.4) we similarly have

$$
z \geq \beta_{k} \geq \frac{1}{k} \sum_{j=1}^{k} \beta_{j} \geq \sqrt{4 \pi} \frac{d}{d+1}\left(\frac{\Gamma\left(1+\frac{d}{2}\right)}{|D|}\right)^{\frac{1}{d}} k^{\frac{1}{d}}
$$

and so

$$
N_{K}(z) \leq \frac{(d+1)^{d}}{d^{d}} \frac{1}{(4 \pi)^{\frac{d}{2}}} \frac{|D|}{\Gamma\left(1+\frac{d}{2}\right)} z^{d} .
$$

Since (4.13) is a cruder upper bound than (4.14) as $(d+1) / d \leq d /(d-1)$, we simply take (4.14) to conclude the proof.

\section{ACKNOWLEDGMENT}

The author would like to thank Selma Yıldırım Yolcu for her valuable comments on a preliminary version of this manuscript. 


\section{REFERENCES}

[1] F. A. Berezin, Covariant and contravariant symbols of operators, Izv. Akad. Nauk SSSR Ser. Mat. 36 (1972), 1134-1167 (Russian). MR0350504(50 \#2996)

[2] R. M. Blumenthal and R. K. Getoor, The asymptotic distribution of the eigenvalues for a class of Markov operators, Pacific J. Math. 9 (1959), 399-408. MR.0107298 (21 \#6023)

[3] Evans M. Harrell II and Selma Yıldırım Yolcu, Eigenvalue inequalities for Klein-Gordon operators, J. Funct. Anal. 256 (2009), no. 12, 3977-3995, DOI 10.1016/j.jfa.2008.12.008. MR2521917(2010e:35198)

[4] Peter Li and Shing Tung Yau, On the Schrödinger equation and the eigenvalue problem, Comm. Math. Phys. 88 (1983), no. 3, 309-318. MR701919 (84k:58225)

[5] Elliott H. Lieb and Michael Loss, Analysis, 2nd ed., Graduate Studies in Mathematics, vol. 14, American Mathematical Society, Providence, RI, 2001. MR.1817225 (2001i:00001)

[6] Antonios D. Melas, A lower bound for sums of eigenvalues of the Laplacian, Proc. Amer. Math. Soc. 131 (2003), no. 2, 631-636 (electronic), DOI 10.1090/S0002-9939-02-06834-X. MR.1933356 (2003i:35218)

[7] Selma Yildirim Yolcu, An improvement to a Berezin-Li-Yau type inequality, Proc. Amer. Math. Soc. 138 (2010), no. 11, 4059-4066, DOI 10.1090/S0002-9939-2010-10419-7. MR2679626(2011m:35255)

[8] S. Yıldırım Yolcu and T. Yolcu, Estimates on the eigenvalues of the clamped plate problem on domains in Euclidean spaces, J. Math. Phys. 54 (2013), no. 4, 043515, DOI $10.1063 / 1.4801446$.

[9] Selma Yildirim Yolcu and Türkay Yolcu, Bounds for the eigenvalues of the fractional Laplacian, Rev. Math. Phys. 24 (2012), no. 3, 1250003, 18, DOI 10.1142/S0129055X12500031. MR2902846

[10] S. Yıldirım Yolcu and T. Yolcu, Estimates for the sums of eigenvalues of the fractional Laplacian on a bounded domain, Comm. Contemp. Math. 15 (2013), no. 3, 1250048, DOI 10.1142/S0219199712500484.

[11] Selma Yıldırım Yolcu and Türkay Yolcu, Multidimensional lower bounds for the eigenvalues of Stokes and Dirichlet Laplacian operators, J. Math. Phys. 53 (2012), no. 4, 043508, 17, DOI 10.1063/1.3701978. MR2953152

[12] G. Wei, H. J. Sun, L. Zeng, Lower bounds for Laplacian and fractional Laplacian eigenvalues, preprint, 2011, http://arxiv.org/abs/1112.4571.

[13] V. Vougalter, Sharp semiclassical bounds for the moments of eigenvalues for some Schroedinger type operators with unbounded potentials, preprint, (2011), http://www.ma. utexas.edu/mp_arc/c/11/11-155.pdf.

Department of Mathematics, Purdue University, West Lafayette, Indiana 47907

E-mail address: tyolcu@math.purdue.edu

Current address: Department of Mathematics, Case Western Reserve University, Cleveland, Ohio 44106

E-mail address: tyolcu@gmail.com 